interventional specialities to try to assess the impact of endoscopy on health. Further exploration of endoscopy workstation and tool design changes may be of benefit to the longterm health of endoscopists.

\section{MUSCULOSKELETAL SYMPTOMS AMONGST ENDOSCOPISTS}

S Burska, B Ryan Department of Gastroenterology, Adelaide and Meath Hospital incorporating The National Children's Hospital, Tallaght, Dublin, Ireland

10.1136/gutjnl-2013-305143.110

Introduction Gastroenterologists may be at risk of overuse / repetitive strain type injuries. The number, complexity and duration of endoscopic procedures, in addition to the career duration are possible contributing factors. Anecdotally and in limited literature, endoscopists report a variety of musculoskeletal problems. Much attention has been paid to the safety of patients during endoscopic procedures, but the safety of the physician is often forgotten.

Aims/Background Firstly to establish the frequency and character of musculoskeletal symptoms in endoscopists and to compare to those in physicians in non-interventional specialties. Secondly to investigate possible contributing factors to these symptoms in endoscopists.

Method Online questionnaires were created which contained 13 simple questions about speciality, age, number, duration and complexity of procedures performed, handedness and other physical activities. Questionnaires were distributed and collected on ISGE meeting and also accessible via the BSG website.

Results 86 responses have been collected to date. Most of them experienced back $(43 \%)$, neck $(36 \%)$ or shoulder pain $(35 \%)$. Physicians perceived that table height and monitor positions were contributors. Musculoskeletal problems were surprisingly not more common in older doctors, who have been scoping for longer. The results from non-interventional specialties are currently awaited and will be available for comparison in the coming weeks.

Conclusion Musculoskeletal symptoms are relatively common amongst endoscopists, and surprisingly were not more common in older, longer practising endoscopists. Our results will be compared to age matched groups of doctors involved in non 